ausgeführten Analysen die Destillate nach Zusatz überschüssiger n.-Natronlauge eingedampft worden waren, wobei ein Anziehen saurer Gase aus der Laboratoriumsatmosphäre nicht leicht völlig ausgeschlossen werden kann, so versuchten wir dieses Eindampfen zu umgehen. Es fragte sich: kann man freie Benzoësäure, in der Verdünnang, wie sie bei den Benzoylbestimmungen direct erhalten wird, mit genügender Gınauigkeit durch $1 / 10^{\circ}$. Barytwasser titriren?

Um hierüber Gewissheit zı erhalten, wurden $1.2134 \mathrm{~g}$ reine Benzo ësäure in Wasser zu $500 \mathrm{ccm}$ gelöst und mit der Lösung folgende Versuche gemacht.

I. $10 \mathrm{ccm}$ Lösung $=0.0243 \mathrm{~g} \quad \mathrm{C}_{7} \mathrm{H}_{6} \mathrm{O}_{9}$ wurdeo auf $100 \mathrm{~cm}$ verdũnnt. Die Titration mit $1 / 10^{-} n$. Barytwasser unter Anwendung von Phenolphtaleĩn ergab $0.0244 \mathrm{~g} \mathrm{C}_{7} \mathrm{H}_{6} \mathrm{O}_{2}$. $\mathrm{C}_{7} \mathrm{H}_{6} \mathrm{O}_{2}$.

2. $50 \mathrm{cem}=0.1213 \mathrm{~g} \mathrm{C}_{7} \mathrm{H}_{6} \mathrm{O}_{2}$, auf $500 \mathrm{ccm}$ verdünnt. gaben $0.1208 \mathrm{~g}$

3. $100 \mathrm{ecm}=0.2+26 \mathrm{~g} \mathrm{C}_{7} \mathrm{H}_{6} \mathrm{O}_{2}$, auf $1000 \mathrm{~cm}$ verdünnt, gaben $0.2441 \mathrm{~g}$ $\mathrm{C}_{7} \mathrm{H}_{6} \mathrm{O}_{2}$.

Die Genauigkeit war demnach binreichend, weshalb die Bestimmuggen dann auch in der vereinfachten Form ausgeführt wurden. In Folge des böberen Molekulargewichtes der Benzoësäure kommen die Analysenfehler im Endergebniss etwas stärker zum Ausdrucke, als bei den Acetylbestimmungen. Trotzdem waren die bei der Untersucbung der Benzoylderirate ron 1.3.6-Trioxynaphtalin und seines Polymerisationsproductes erhaltenen Resultate') für die Zahl der vorhandenen Benzoylgruppen rollkommen entscheidend.

Braunschweig, Technische Hochschule. Labor, für analyt. und techs. Cbemie.

687. Richard Meyer und Hermann Pfotenhauer:

Ueber weohselseitigen Austausch aromatisoher Complexe.

(Kingegangen am 21. November 1905; mitgetheilt in der Sitzung von Hrn. J. Meisenheimer.)

Bei einer Darstellung ron Brenzcatechinphtaleïn ${ }^{2}$ ) sollte das erhaltene Product auf eine etwaige Beimengung von Phtalsäure unteroucht werden, wesbalb eine Probe im Reagensglase mit Resorcin erhitzt wurde. Es resultirte eine braungelbe Schmelze, deren Alkalilösung zwar noch die für Brenzcatechinphtaleïn charakteristische Blau-

1) Vergl. dio voranstehende Abhandlung.

2) Mit einer Untersuchung dieses Körpers sind wir beschäftigt. 
färbung zeigte, zugleich aber lebbaft grüne Fluorescenz. Da indessen Resorcin nicht nur mit Phtalsäure fluorescirende Producte liefert, so konnte der Beweis für die Anwesenheit dieser Säure nicbt als bindend gelten. Andererseits wurde erst vor einiger Zeit gefunden, dass ein Phtaleïn, nämlich Galleïn, mit Phtalsäureanhydrid eine dem Fluore die $>$ Fluoresceïnreactione auch in unserem Falle auf eine andere Ursache als die Condensation von Phtalsäure und Resorcin zurückzuführen war.

Un der Sache näher zu kommen, wurde zunächst im Reagensrohr reines Phenolphtaleïn mit Resorcin zum Kochen erbitzt. Auch hier erhielten wir Fluoresceïnreaction, während andererseits die rothe Farbe der alkalischen Phenolphtaleïn-Lösung nicht zum Verschwinden zu bringen war. Der Versuch wurde darauf mit unzweifelhaft reinem Brenzcatechinphtaleîn wiederholt. Das Ergebniss war dasselbe wie bein ersten Mal. Als aber Hydrochinonphtaleïn mit Resorcin geschmolzen wurde, blieb die Fluoresceïnreaction aus, und die alkalische Lösung der Scbmelze zeigte unverändert die für das Hydrochinonphtaleïn charakteristische Violettfärbung.

Die nähere Untersuchung hat gezeigt, dass beim Verschmeizen son Phenolphtaleïn und Breurcatechiophtaleön mit Resorcin in Wabrbeit Fluoresceïn entsteht. Dabei müssen 2 Phenol-, bezw. Brenzeatechin-Reste durch je 2 Resorcinreste ersetzt werden, unter gleichzeitiger Abspaltung von Wasser und Schliessung des Pyronringes:

$$
\begin{aligned}
& \begin{array}{l}
\mathrm{C}_{6} \mathrm{H}_{4} \cdot \mathrm{C}<\mathrm{C}_{6} \mathrm{H}_{4} \cdot \mathrm{OH} \\
\mathrm{C}_{\mathrm{O}-\mathrm{O}}
\end{array}
\end{aligned}
$$

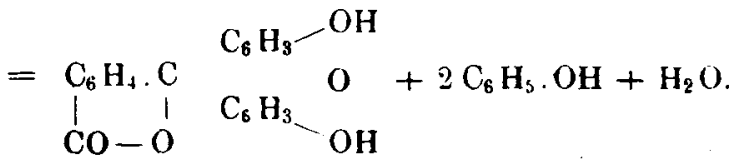

$$
\begin{aligned}
& \begin{array}{l}
\mathrm{C}_{6} \mathrm{H}_{4} \cdot \mathrm{C}-\mathrm{C}_{6} \mathrm{H}_{3}(\mathrm{OH})_{2} \\
\mathrm{CO}-\mathrm{O}
\end{array}
\end{aligned}
$$

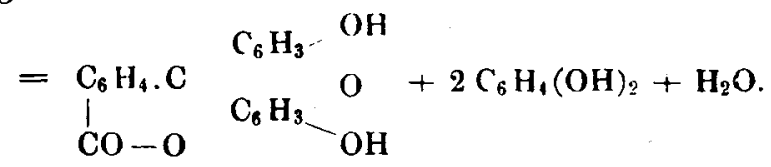

In der That konnte bei dem zweiten Vorgange die Abspaltung rnn Brenzcatechin nachgewiesen werden.

3) R. Meyer, diese Berichte 36, 1561 [1903]. 
Das Hydrochinonphtalë̈n unterscheidet sich von den beideu anderen Phtaleïnen dadurch, dass es deu Pyronring bereits enthält. Es konnte hiernach kaum bezweifelt werden, dass dieses Atomgebilde bei den beobachteten Reactionen eine entscheidende Rolle spielt. Die Anwesenbeit des Pyronringes erhöht die Stabilität des Moleküls, und die Entstehung der beständigeren Verbindung aus den weniger beständigen erscheint $\mathrm{rom}$ Standpunkte der ehemischen Statik einigermaassen verständlich. Es liegt dann freilich die Folgerung nahe, dass diese Umsetzungen umkehrbar sein sollten. Die Thatsache, dass Phenolphtaleïn nicht vollständig in Fluoresceïn übergeführt werden konnte, welche sich auch bei den Versuchen in grösserem Maassstabe bestätigte, schien in der That auf den Eintritt eines Gleichgewichtes hinzudeuten. Aber einerseits gelang es beim Brenzcatechinphtaleïn, die vollständige Umsetzung berbeizuführen; andeserseits zeigte eiu directer Versuch, dıss fertiges Fluoresceïn auch bei lang andauerndem Erhitzen mit einem grossen Ueberschuss an Phenol keine Spur von Phenolphtalein liefert. Eine Umkehrbarkeit des Processes konote also durch den Versuch nicht nachgewiesen werden.

Der Austausch von an Koblenstoff gebundenen, aromatischen Complexen gegen andere Complexe derselben Art ist unseres Wissens in dieser Weise noch nicht beobachtet. Es erscheint aber nicht unmöglich, den festgestellten Thatsachen noch eine etwas andere Deutung zu geben. Mau könnte sicb vorstellen, dass nicht der Pbenoloder Brenzcatechin-Rest als solcher gegen den Resorcintest ausgetauscbt wird, sondern nur die Hydroxylgruppen des Brenzcatecbink, oder beim Phenolphtalein die Hydroxylgruppen und je ein Kernwasserstoffatom gegen die Hydroxylgruppen des Resorcius. Danach würde die Umsetzung beim Phenolphtaleïn entweder im Sinne des folgenden Schema I oder aber nach II verlaufen:

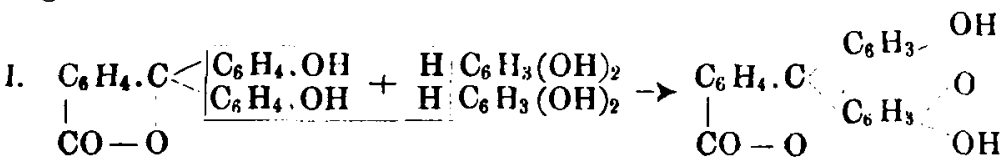

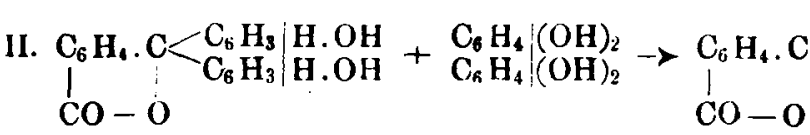

$$
\begin{aligned}
& \mathrm{C}_{n_{3}} \mathrm{H}_{3}-\mathrm{OH} \\
& \mathrm{C}_{6} \mathrm{H}_{3} \quad \mathrm{O}
\end{aligned}
$$

Die zweite Auffassung erscbeiut vielleicht wenig wahrscheinlich, jedoch ganz unberücksichtigt darf sie wohl nicht bleiben. Da man aber in beiden Fällen dasselbe Fluoresceïn erbält, so lässt sich ohne weiteres $z$ wischen I und II nicht entscheiden. Eine Möglichkeit hierzu würde sich bieten, wenn man die in Betracht kommenden 
Phenolcomplexe durch Einführung substituirender Gruppen gewissermaassen zeichuete, wie es gelegentlich bei äbnlichen Fragen geschehen ist. Würde man z. B. Phenol- oder Brenzcatechin. Phtaleĩn statt mit Resorcin mit 2.4-Dibromresorcin verschmelzen, so müsste man, im Falle eines glatten Verlaufes der Reaction, nach I Eosin erhalten, nach II dagegen wieder Fluoresceïn. Wir konnt'n bisher nur einen Reagensglasversuch in dieser Richtung anstellen, nach dessen Ausfall in der That Eosin zu entstehen scheint. Die Frage muss aber noch eingebender nntersucht werden, womit wir eben beschäftigt sind. Dabei wird zugleich auf die Möglichkeit Räcksicht zu nehmen sein, dass vielleicht die Reactionen I uud II neben einander verlaufen; in diesem Falle müssten Eosin und Fluoresceïn zugleich gebildet werden. Selbstrerständlich könnte man die Zpichnung des Resorcinrestes auch durch andere Substituenten bewirken und beispielsweise Phenolphtaleïn mit Orcin verschmelzen, u. s. f.

Die Reaction, über welche hier berichtet wird, scheint einer weiteren Ausdebnung fäbig zu sein. Einige orientirende Versuche haven gezeigt, dass $p$-Rosanilin, Aurin, Michler'sches Keton etc. beim Verschmelzen mit Resorcin, sowie auch mit den alkylirten $m$-Aminophenolen gleichfalls in andere, meist fluorescirende Verbindongen übergeführt werden. Wir behalten uns vor, über diese Umsetzungen weiter zu berichten. Vorläufig sei nur erwähnt, dass beim Erhitzen von $p$-Rosanilin mit Resorcin die Abspaltung von Auilin nachgewiesen wurde.

\section{Specieller Theil.}

1. Phenolphtaleĩu und Resorcin. $5 \mathrm{~g}$ Phenolphtaleĩn und $15 \mathrm{~g}$ Resorcin wurden im Oelbade anf $180-200^{\circ}$ erhitzt, und von Zeit zu Zeit durch Glasstabproben, welche in Natronlauge gebracht wurden, der Fortgang der Umsetzung beobachtet. Die Operation wurde so lange in Gang erbalten. bis die Fluorescenz die Rothfärbung bedeutend übertraf, beispielsweise 48 Stunden. Auch mit einem grösseren Ueberschuss an Resorcin konnte vollständige Umsetzung nicht erreicht werden. Es wurde nun das unveränderte Resorcin mit dem abgespaltenen Phenol im Vacuum abdestillirt; als dann die Schmelze noch heiss aus dem Gefässe gebracht wurde, erkaltete sie zu einer spröden, gelbbraunen Masse. Es gelang nicht, aus dieser reives Fluoresceïn zu gewinuen, auch die Acetylirung und Benzoylirung des Productes fühıten vicht zu reinen Körpern. Mit besserem Erfolge versuchten wir das entstandene Fluores ce in als Eosin zu charakterisiren. Za diesem $Z$ wecke wurde die nach dem Abtreiben der Phenole zurückgebliebene Masse gepulvert, in Alkohol gelöst und dann so lange Brom hinzugetropft, bis ein bleibender intensirer Bromgeruch das 
Ende der Reaction anzeigte. Die dunkelbranne Farbe der Lö8ung schlug dabei in Rothbraun um. Nach mehrstündigem Stehen wurde die Lösung in Wasser gegossen, wodurch das Roh-Eosin zunächst als scbmutzig braune Masse ausfiel. Es wurde abfiltrirt, getrocknet, in Alkohol gelöst, die Lösung mehrere Stunden mit Thierkoble gekocht und diese Operationen noch zwei Mal wiederbolt. Das so erhaltene Product war $z$ war noch nicht rein genug, um es zum Krystallisiren zu bringen, es gab sich aber schon durch die Farbe una Fluorescenz seiner Lösungen als Losin zu erkennen. Die Seidenfaser färbte es in der für Eosin charakteristischen Weise. Durch Natriumamalgam wurde die alkalische Lösung unter Bildung von Fluorescin entfärbt; auf Zusatz ron Ferricyankalium trat dann sofort intensive Flnoresceïnreaction ein.

Der bestimmte Nachweis, dass Eosin vorlag, wurde durch Umwandlung in das A cetat gefübrt. Das aus dem Rob-Eosin mittels Essigsäureanbydriu erbaltene und in der üblichen Weise isolirte Product wurde zuerst durch längeres Kochen der alkoholischen Lösung mit Thierkohle, darauf durch mehrmaliges abwechselndes Umkrystallisiren aus Benzol und Chloroform gereinigt. Es wurde so in schwach-röthlichen Kryställchen erbalten.

$0.0206 \mathrm{~g}$ Sbst.: $0.07: 0 \mathrm{~g} \mathrm{\Delta gBr}$. $\mathrm{C}_{20} \mathrm{H}_{6} \mathrm{O}_{5} \mathrm{Br}_{4}\left(\mathrm{C}_{2} \mathrm{H}_{3} \mathrm{O}\right)_{2}$. Ber. $\mathrm{Br}$ 43.7. Gef. Br 43.3.

Du bisher von dem Eosinacetat noch keine Analyse vorlag, so haben wir auch aus käuflichem Eosin das Acetat dargestellt.

$0.1178 \mathrm{~g}$ Sbst.: $0.1210 \mathrm{~g} \mathrm{AgBr}=43.7 \mathrm{pCt}$.

Beide von uns dargestellten Acetate stimmen in ihren Eigenschaften genau überein. Bei etwa $180^{\circ}$ fangen sie an, sich rosa zu färben; die Farbe wird bei weiterem Erwärmen dunkler, und gegen $290^{\circ}$ schmelzen sie unter Zersetzang ${ }^{1}$ ).

2. Brenzcatechinphtaleïn and Resorcin. $1 \mathrm{~Tb}$. des Phtaleïns wurde mit $5 \mathrm{~T}$. Kesorcin im Oelbade auf $210-220^{\circ}$ erbitzt. Schon uach einer balben Stunde zeigte eine Probe in Alkali neben der blanen Brenzcatechinphtaleïnfarbe grüne Fluorescenz; allmählich trat die blaue Farbe mehr und mehr zarück und war nach einigen Stunden vollständig verschwunden. In diesem Falle konnte das Reactionsproduct direct au Fluoresceïnacetat rerarbeitet werden, welches darch mehrfaches Umkrystallisiren aus Alkohol tein erbalten wurde. Scbmp. $200^{n 2}$ ).

$0.2308 \mathrm{~g}$ Sbst.: $0.5908 \mathrm{~g} \mathrm{CO}_{2}, 0.0822 \mathrm{~g} \mathrm{H}_{2} \mathrm{O} .-0.2310 \mathrm{~g}$ Sbst.: $0.5933 \mathrm{~g}$ $\mathrm{CO}_{2}, 0.0796 \mathrm{~g} \mathrm{H}_{2} \mathrm{O}$.

3) Vergl. Ad. Baeyer, Ann d. Chem 183, 53 [1876].

2) Id. ib. 13. 


$$
\mathrm{C}_{20} \mathrm{H}_{10} \mathrm{O}_{5}\left(\mathrm{C}_{2} \mathrm{H}_{3} \mathrm{O}\right)_{2} . \begin{aligned}
& \text { Ber. O 69.2, } \\
& \text { Gef. 》 } 69.8,69.1, \text { - 3.9, 3.8. }
\end{aligned}
$$

Un den Nachweis zu fübren, dass bei der Reaction Brenzcatechin abgespalten wird, destillirten wir $1 \mathrm{~g}$ Brenzcatechinphtaleïn und 5 g Resorcin aus einer kleinen Retorte. Das Destillat erstarrte krystallinisch; der Rückstand zeigte sehr starke Fluoresceïreaction, dagegen nicht mehr die Reaction auf Brenzcatechinphtalein. Um Spuren übergerissener Pbtaleïne aurückzubalten, wurde das Destillat nocb ein Mal umdestillirt. Die zuerst übergegangenen Antbeile wurden in Wasser gelöst, von einer Trübung abfiltrirt und mit Natronlauge rersetzt. worauf die für Brenzcatechin charakteristische Braunfärbnng eintrat, welche ron der Oberfläche ausging und sich bei längerem Stehen durch die ganze Flüssigkeit verbreitete.

3. Hydrochinonphtalein uod Resorcin. 1 Th. Hydrochinonphtaleïn und $5 \mathrm{Th}$. Resorcin wurden am Rückflusskübler im Oelbade 12 Stunden lang auf $200-220^{\circ}$ erhitzt. Es konnte keinerlei lmsetzung beobachtet werden: Die genommenen Proben zeigten in Alkali stets die für Hydrochinonphtaleïn cbarakteristische Violettfärbung, ohne jede Fluorescenz. Nur wurde die Schmelze allmählich dunkler und die Hydrochinonphtaleïnreaction unreiner.

4. Fluoresceïn und Phenol. $1 \mathrm{~g}$ Fluoresceïn und $12 \mathrm{~g}$ Phenol wurden 12 Stunden lang am Rückflusskühler auf freier Flamme zum Sieden erhitzt. Die Alkaliprobe zeigte in keinem Stadium die geringete Rothfärbung, sondern unverändertes Bestehen der Fluoresceïnreaction.

Braunschweig. Techn. Hochschule. Laboratorium für analyt. und techn. Chemie.

688. R. Kempf: Oxydationen mit Silberperoxyd.

\section{Die Oxydation von Oxalsäure.}

[Aus dem I. chem. Institut der Universitat Berlin.]

(Eingegangen am 29. November 1905.)

Damit beschäftigr, die Einwirkung von Metallsuperoxyden auf organische Substanzen in saurer Lösung zu untersucben, gelangte ich zu dem Resultat, dass ron den angewendeten Superoxyden -- u. a. Blei-, Kobalt-, Mangan- und Silber-Superoxyd - sich ganz besonders das zuletzt genannte durch eine äusserst energische Oxydationskraft auszeichnet. So führt Silbersuperoxyd z. B. Benzol bei Gegenwart von Salpetersäure momentan unter freiwilliger Erwärmung in Benzo- 